\title{
What Leads to a Patient Refusal for Ambulatory Surgery? A Logistic Regression Prediction Model Based on a Five Year Retrospective Analysis of Patients with Abdominal Wall Hernia
}

\author{
O que Leva à Recusa de um Doente Para Cirurgia de \\ Ambulatório? Um Modelo Preditivo de Regressão Logística \\ Baseado numa Análise Retrospetiva de Cinco Anos de Doentes \\ com Hérnia da Parede Abdominal
}

\author{
João OLIVEIRA $\rrbracket^{1}$, Sandra F. RAMOS ${ }^{2,3}$, Manuel B. CRUZ², Isabel NOVAIS ${ }^{1,4}$, Carlos MAGALHÃES ${ }^{4,5}$, \\ Marisa SANTOS $1,4,6$ \\ Acta Med Port 2022 Mar;35(3):184-191 - https://doi.org/10.20344/amp.15733
}

\section{ABSTRACT}

Introduction: Ambulatory surgery has proven benefits in patient wellbeing and cost reduction in healthcare systems. However, some patients referred for ambulatory surgery are refused and directed instead towards inpatient care, which generates several drawbacks. The reasons for this refusal have not been yet studied. The aim of this study is to identify, retrospectively, significant variables associated with patient refusal for ambulatory surgery and develop a mathematical tool able to predict with strong accuracy those who will be rejected.

Material and Methods: Over a 5-year period (2014 - 2018), all patients that underwent abdominal hernia repair in our hospital in an inpatient setting, and that had been previously refused for ambulatory surgery, were analysed for a total of 94 variables. A multivariate logistic regression model was developed to identify risk factors associated with refusal using data from 136 patients (65 refused vs 71 accepted). A prediction index for refusal in ambulatory surgery (IRAS), was derived and tested ( $\mathrm{n}=62$ patients).

Results: The risk index included five significant risk factors: type 2 diabetes mellitus [OR $14.669(2.982 ; 72.154)$ ], physical status [OR 49.155 (15.532; 155.555)], prior malignancy [OR 14.518 (2.653; 79.441)], prior abdominal surgery [OR 3.455 (1.006; 11.866)] and usage of antiplatelet agents [OR 25.600 (4.309; 152.066)]. All risk factors were associated with a high risk of refusal (OR between 3.455 for history of prior abdominal surgery and 49.155 according to the American Society of Anaesthesiologists physical status classification). Defining five points as the maximum IRAS score that predicts suitability for ambulatory surgery resulted in a positive predictive value of $93.55 \%$ and negative predictive value of $87.10 \%$.

Conclusion: IRAS is a useful tool that can contribute to reduce time to surgery and improve patients' quality of life.

Keywords: Ambulatory Surgical Procedures; Logistic Model; Patient Selection

\section{RESUMO}

Introdução: A cirurgia de ambulatório tem benefícios comprovados no bem-estar dos doentes e na redução de custos dos sistemas de saúde. Porém, alguns doentes referenciados para cirurgia de ambulatório são recusados e encaminhados para internamento. Os motivos desta recusa ainda não foram estudados. Neste trabalho identificámos, retrospectivamente, variáveis significativas na recusa dos doentes e fornecemos uma ferramenta matemática capaz de prever de forma precisa aqueles que serão rejeitados.

Material e Métodos: Ao longo de cinco anos (2014 - 2018), todos os doentes submetidos a correção cirúrgica de hérnia abdominal em regime de internamento no nosso centro hospitalar previamente recusados para cirurgia de ambulatório foram analisados para um total de 94 variáveis. Um modelo de regressão logística multivariada foi desenvolvido para identificar os fatores de risco para recusa usando dados de 136 doentes (65 recusados vs 71 aceites). Um índice preditivo para recusa de cirurgia em ambulatório (IRAS), foi criado e testado ( $n=62$ doentes).

Resultados: O IRAS incluiu cinco fatores de risco significativos: diabetes mellitus tipo 2 [OR 14,669 (2,982; 72,154)], estado físico [OR 49,155 (15,532; 155,555)], neoplasia maligna prévia [OR 14,518 $(2,653 ; 79,441)]$, cirurgia abdominal prévia [OR 3,455 (1,006; $11,866)]$ e uso de agentes antiplaquetários [OR 25,600 $(4,309 ; 152,066)]$. Todos os fatores de risco foram associados a elevado risco de recusa (OR entre 3,455 para história de cirurgia abdominal prévia e 49,155 de acordo com a classificação do estado físico segundo a American Society of Anaesthesiologists). A definição de cinco pontos como a pontuação máxima do IRAS que prevê adequação para cirurgia de ambulatório resultou num valor preditivo positivo de $93,55 \%$ e um valor preditivo negativo de $87,10 \%$.

Conclusão: O índice IRAS é uma ferramenta útil que pode contribuir para a redução dos tempos de espera e melhorar a qualidade de vida dos doentes.

Palavras-chave: Modelos Logísticos; Procedimentos Cirúrgicos Ambulatórios; Selecção de Doentes

1. Serviço de Cirurgia Geral. Centro Hospitalar Universitário do Porto. Porto. Portugal.

2. Laboratório de Engenharia Matemática. Instituto Superior de Engenharia do Porto. Instituto Politécnico do Porto. Porto. Portugal.

3. Centro de Estatística e Aplicações. Universidade de Lisboa. Lisboa. Portugal.

4. Instituto de Ciências Biomédicas Abel Salazar. Universidade do Porto. Porto. Portugal.

5. Centro Integrado de Cirurgia de Ambulatório. Centro Hospitalar Universitário do Porto. Porto. Portugal.

6. Unidade de Cirurgia Colorretal. Centro Hospitalar Universitário do Porto. Porto. Portugal.

$\triangle$ Autor correspondente: João Oliveira. joao.oliveira@chporto.min-saude.pt

Recebido: 14 de janeiro de 2021 - Aceite: 12 de maio de 2021 - First published: 19 de julho de 2021 - Online issue published: 02 de março de 2022

Copyright $\odot$ Ordem dos Médicos 2022 


\section{INTRODUCTION}

Ambulatory surgery has grown in developed countries in the last 50 years and it has increasingly become more established in developing countries as well..$^{1-3}$ It is considered a safe, efficient and cost-effective level of care contributing to patient satisfaction and improved quality of life. ${ }^{4-6}$ Patients initially proposed for ambulatory surgery across the different medical specialties can be rejected and redirected to inpatient care. This imposes a delay in the resolution of the patients' condition with added days of morbidity, increased loss of both working days and healthcare costs.

Refusal of a given patient is a medical decision and relies mostly on anaesthesiologists or surgeons that conduct the preliminary medical assessment. Although efforts have been made to conduct a pre-operative assessment and patient preparation prior to ambulatory surgery, there are no defined guidelines to reject a patient based on his/her clinical information. ${ }^{7}$

A tool that can predict with a reasonable amount of accuracy which patients will be accepted or refused for surgery in the outpatient setting, can be an important aid for the initial referral by both primary care specialties or other medical specialties that can also refer patients. In this way, the clinician would be more supported and empowered in deciding if the initial referral should be made to either outpatient or inpatient care, which is beneficial to both the patient and the healthcare system. The possibility to take a more informed decision would deliver quicker and safer solutions to all patients, thus decreasing the burden of disease and improving the clinical management.
The aim of this study is to propose a statistical methodology to identify which variables best predict whether a patient is likely to be rejected or accepted for ambulatory surgery and to develop a risk score to predict the refusal of a given patient by using a registry of patients that have been referred for ambulatory surgery.

\section{MATERIAL AND METHODS Database and study population}

A retrospective observational study was conducted at our centre from the $1^{\text {st }}$ January 2014 to $31^{\text {st }}$ December 2018 (five years) in accordance with the specifications and approval from the local ethics committee. During this period, all the adult patients (age $\geq 18$ years) undergoing elective abdominal wall hernia surgery in inpatient care were analysed 1593 patients). The authors implemented a 1:1 study consisting of an equal size between refused and non-refused patients for ambulatory surgery.

Based on the clinical records, those that were initially proposed for ambulatory surgery and refused, and ultimately undergoing surgery in inpatient care (78 patients), were selected for further analysis. Among these, 13 patients were excluded due to social reasons and were rejected from further analysis. Therefore, a total of 65 patients were included in our study sample in the refusal group.

Among the group of patients that underwent elective abdominal wall hernia surgery in the ambulatory setting during the same 5-year period, a control group ( $n=71$ patients) were selected by random number generation.

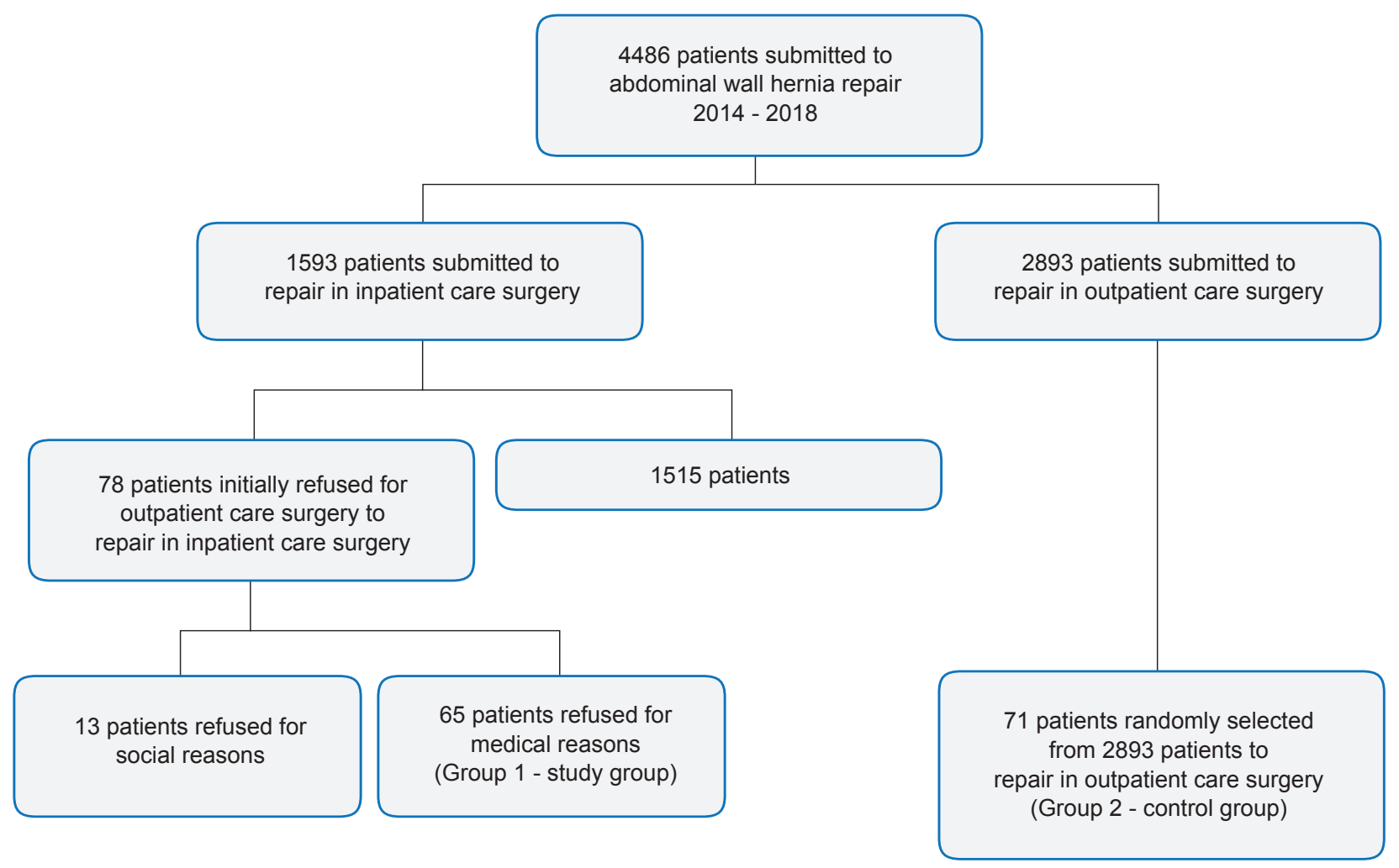

Figure 1 - Flow diagram from the initial time of patient selection to the composition of the final patient samples 
Fig. 1 shows the flow diagram from the initial time of patient screening to the establishment of the final patient sample.

A total of 94 parameters, which included data on comorbidities, daily habits, anthropometry, prior surgical record, anaesthetic complications, and other considered relevant were collected from the patient medical record and clinical registries, in both the study and control groups. Additionally, both the reason and the medical specialty responsible for refusal of ambulatory surgery in the study group were gathered.

The reasons for refusal of the 78 patients that had initially been proposed for surgery in the ambulatory setting, and subsequently redirected to inpatient care, were scrutinised. Thirteen patients $(16.7 \%)$ were rejected due to social reasons, mainly associated with a considerable degree of dependency on others for the daily tasks, in patients that lived alone or that had reduced home support. These patients were excluded from further analyses. Sixty-five (83.3\%) patients [50 males $(76.9 \%)$ ] were rejected after being evaluated by the anaesthesiologist $(81.9 \%)$ or the surgeon $(18.1 \%)$ and these patients constituted our study group (Group 1)].

The reasons for refusal due to medical reasons were gathered through analysis of the medical records and are presented in Fig. 2. A threshold of $5 \%$ was defined, and only reasons that were represented above this limit are shown. In this sense, those who represented more than 5\% were: cardiac conditions $(21.8 \%)$, respiratory conditions $(19.2 \%)$, obstructive sleep apnoea (13.9\%), cardiovascular risk factors $(9.9 \%)$, difficult orotracheal intubation $(7.7 \%)$, previous post-operative hemodynamic instability $(6.8 \%)$ and chronic kidney disease (5.8\%). The hernia size was the single rea- son specified by the surgeon for patient rejection for ambulatory surgery, with the remaining reasons being attributed to the anaesthesiologist.

Afterwards, and for each patient refused, the selected 94 variables were analysed and coded.

By means of a random number generator, a random sample of 71 patients among the population of adult patients undergoing elective abdominal wall hernia surgery in outpatient care from the $1^{\text {st }}$ January 2014 to $31^{\text {st }}$ December 2018 ( $n=2893$ patients) was selected. This group of patients Group 2, was analysed and coded for the same variables chosen for those refused for ambulatory surgery.

\section{Statistical analysis}

A multivariate logistic regression model was developed to predict refusal in ambulatory surgery using a training-test strategy. Logistic regression models can be used to identify potential risk factors for refusal of ambulatory surgery. The patient's total risk index is the result of a simple linear function of the risk factors, which are previously quantified and weighed.

In order to identify and remove features with little or no predictability of the target to prevent overfitting and to identify highly correlated or redundant features while suppressing the negative impacts towards the model without losing important information, the first stage of the proposed methodology consisted of a bivariate analysis to investigate the presence of a relationship between refusal and each potential risk factor based on the hospital's training data. In this bivariate analysis, whenever possible, the chi-square test or Fisher's exact test (only for $2 \times 2$ contingency tables) for nominal variables and the $t$ test or the Z-test for

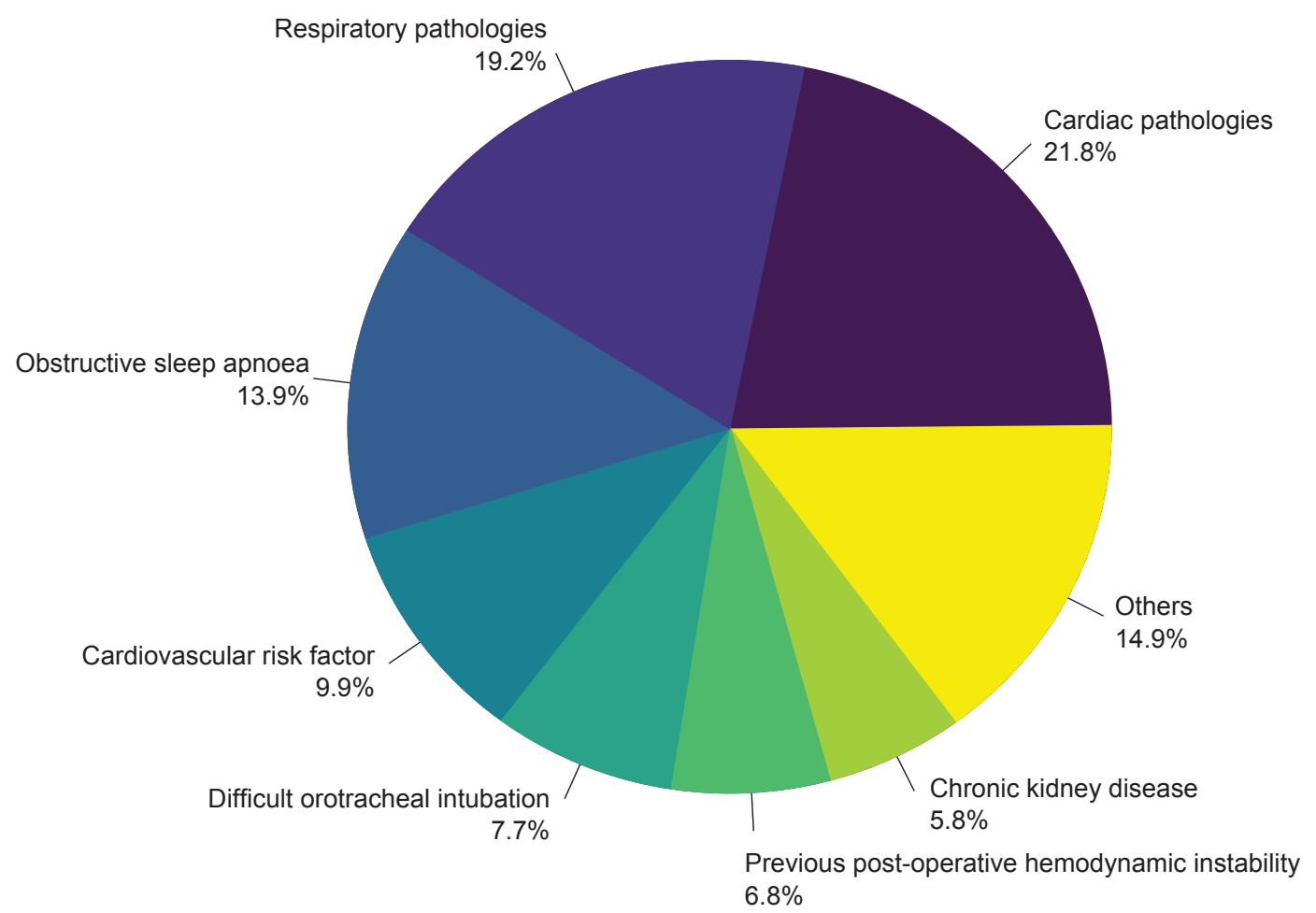

Figure 2 - Medical reasons presented for refusal of patients referred to ambulatory surgery 
continuous variables were applied.

The potential risk factors examined included comorbidities, daily consumption of alcohol and tobacco habits, anthropometric indicators, prior surgical record, anaesthetic complications, and other relevant considerations. The risk factors that were significantly related with refusal of ambulatory surgery were considered as a natural candidate for independent variables in the multivariate logistic regression model.

In order to identify which variables best predicted whether a patient was likely to be rejected, a multivariate logistic regression model with stepwise forward variable selection method was then estimated. All variables with $p<0.10$ were included in the final model. The discrimination power of the final predictive model was evaluated with the $\mathrm{C}$-statistic (also called as area under the receiver operating characteristic curve, AUC) and the Hosmer-Lemeshow test was used to evaluate the goodness of the fit (calibration).

The third step of the proposed methodology included the prediction index for refusal in ambulatory surgery (IRAS) derivation. This index was developed as a score system that is easy to use and that requires simple calculations. IRAS is given by the sum of points assigned to each category of the significant risk factors. The point value for the $j$-th category of the $i$-th risk factor was defined as the ratio between the respective regression coefficient, $\beta_{i, j^{\prime}}$ and its standard error $s e_{i, j}$ (normalization), and this ratio was rounded to the nearest integer.

Finally, the risk score was validated using a test group (n $=62$ patients) - validation cohort - consisting of patients that underwent elective abdominal wall hernia surgery in random time points during 2019, in either inpatient or ambulatory setting.
All the data analysis was performed in R, version 3.5.2 (Foundation for Statistical Computing, Vienna, Austria).

\section{RESULTS}

Among the candidate predictors, based on 94 different clinical parameters, there are 15 significant risk factors. Table 1 presents the univariate frequencies and prevalence (\%) for all significant candidate predictors in both groups. Data is presented as frequencies (prevalence in percentage) for categorical variables and as mean \pm standard deviation for the age variable.

\section{Multivariable analysis}

The 15 significant risk factors identified in bivariate analysis were considered as potential predictors in a multivariate logistic regression model, with stepwise forward variable selection method.

Table 2 presents the results of a logistic regression model that was developed to predict refusal for ambulatory surgery.

From these 15, and using a stepwise strategy, only five were considered significant namely, the presence of type 2 diabetes mellitus, presence of higher physical status classification according to the American Society of Anaesthesiologist [(ASA) 3/4], history of prior malignancy, history of prior abdominal surgery and usage of antiplatelet agents. All risk factors were associated with a very high risk of refusal for ambulatory surgery (odds ratio - OR - range between 3.455 for history of prior abdominal surgery and 49.155 for the presence of higher physical status classification ASA 3/4). The predictive performance of the refusal prediction model was assessed by calibration and discrimination. The model fit the data well in terms of discrimination $(A \cup C=0.806)$ and

Table 1 - Significant risk factors for refusal of ambulatory surgery

\begin{tabular}{|c|c|c|c|}
\hline Risk factor & $\begin{array}{l}\text { Group } 1 \\
(n=65)\end{array}$ & $\begin{array}{l}\text { Group 2 } \\
(n=71)\end{array}$ & $p$ value* \\
\hline Age (years) & $65.50 \pm 12.93$ & $53.86 \pm 11.75$ & $<0.001$ \\
\hline High blood pressure & $37(57.0)$ & $16(23.0)$ & $<0.001$ \\
\hline Dyslipidemia & $31(48.0)$ & $11(15.0)$ & $<0.001$ \\
\hline Type 2 diabetes mellitus & $18(28.0)$ & $3(4.0)$ & $<0.001$ \\
\hline Atrial fibrillation & $9(14.0)$ & $1(1.0)$ & 0.007 \\
\hline Obstructive sleep apnoea under Ci-PAP & $11(17.0)$ & $1(1.0)$ & 0.002 \\
\hline Chronic kidney disease & $9(14.0)$ & $0(0.0)$ & 0.001 \\
\hline History of prior malignancy & $12(18.0)$ & $3(4.0)$ & 0.013 \\
\hline Prior abdominal surgery & $22(34.0)$ & $11(15.0)$ & 0.012 \\
\hline Previous acute coronary syndrome & $6(9.0)$ & $0(0.0)$ & 0.028 \\
\hline Cardiac ischemia & $13(20)$ & $0(0.0)$ & $<0.001$ \\
\hline Chronic obstructive pulmonary disease & $14(21.2)$ & $2(2.7)$ & $<0.001$ \\
\hline Severe physical status classification (ASA 3/4) & $56(86.0)$ & $9(13.0)$ & $<0.001$ \\
\hline Usage of hipocoagulants & $9(14.0)$ & $1(1.0)$ & 0.008 \\
\hline Usage of antiplatelet agents & $27(42.0)$ & $2(3.0)$ & $<0.001$ \\
\hline
\end{tabular}

Group 1: refused patients; Group 2: non-refused patients.

ASA: American Society of Anesthesiologists; ASA $3 / 4$ : severe physical status (class 3 and class 4 ).

${ }^{*}$ With the exception of the age variable, whose $p$-value is associated with a t-test, the remaining p-values are associated with the chi-square test of independence. 
Table 2 - Logistic regression model to predict refusal of ambulatory surgery.

\begin{tabular}{lccc}
\hline Risk factor & Coefficient & OR [90\% Cl] & p value \\
\hline Type 2 diabetes mellitus & 2.686 & $14.669[2.982,72.154]$ & 0.006 \\
Severe physical status classification (ASA 3/4) & 3.895 & $49.155[15.532,155.555]$ & $<0.001$ \\
History of prior malignancy & 2.654 & $14.518[2.653,79.441]$ & 0.009 \\
Prior abdominal surgery & 1.240 & $3.455[1.006,11.866]$ & 0.098 \\
Usage of antiplatelet agents & 3.243 & $25.600[4.309,152.066]$ & 0.003 \\
\hline
\end{tabular}

Table 3 - Score associated with refusal of ambulatory surgery

\begin{tabular}{lcc}
\hline Risk factor & Category & IRAS \\
\hline Type 2 diabetes mellitus & No (reference) & Yes \\
Severe physical status classification (ASA 3/4) & No (reference) & 3 \\
& Yes & 0 \\
History of prior malignancy & No (reference) & 0 \\
& Yes & 3 \\
Prior abdominal surgery & No (reference) & 0 \\
Usage of antiplatelet agents & Yes & 2 \\
\hdashline Risk index & 0 (reference) & 0 \\
\hline
\end{tabular}

calibration ( $p=0.113$ in Hosmer-Lemeshow goodness-of-fit test).

Refusal probability for ambulatory surgery using IRAS

Depending on the power of the selected predictors measured by regression coefficients and corresponding precision, an index for refusal in ambulatory surgery (IRAS) was developed as a score system that is easy to use and that requires simple calculations. All factors included in the index are categorical with two categories. The point value for the $i$-th risk factor was defined as the ratio between the regression coefficient, $\beta_{i}$, and its standard error (normalization), and this ratio was rounded to the nearest integer.

Table 3 and Appendix 1 (see Appendix 1: https://www. actamedicaportuguesa.com/revista/index.php/amp/article/ view/15733/Appendix_01.pdf) present the point values for all significant risk factors identified in the logistic regression model. Excluding reference categories, which were given zero points, point values ranged from two for the history of prior abdominal surgery to six for the presence of severe physical status classification (ASA 3/4). The index IRAS

Table 4 - Risk estimation (\%) associated with refusal of ambulatory surgery

\begin{tabular}{cccc}
\hline $\begin{array}{c}\text { IRAS } \\
\text { score }\end{array}$ & $\begin{array}{c}\text { Estimated } \\
\text { risk }\end{array}$ & $\begin{array}{c}\text { IRAS } \\
\text { score }\end{array}$ & $\begin{array}{c}\text { Estimated } \\
\text { risk }\end{array}$ \\
\hline 0 & 3.855 & 9 & 95.095 \\
2 & 13.672 & 11 & 98.711 \\
3 & 23.939 & 12 & 99.347 \\
5 & 55.419 & 14 & 99.833 \\
6 & 71.185 & 15 & 99.916 \\
8 & 90.704 & 17 & 99.979 \\
\hline
\end{tabular}

ranges from 0 to 17 points, 0 for a patient without any risk factors and 17 for a patient with all the five risk factors.

In order to estimate a probability of refusal $(\hat{p})$, a new univariate logistic model was adjusted using the value of the IRAS index as predictor and the refusal (binary variable) variable as dependent variable. The estimated logistic regression equation may be written as:

$$
\hat{p}=\frac{1}{1+e^{(-3.216+0.687 \times I R A S)}}
$$

where -3.216 is the intercept of the univariate logistic regression model and 0.687 is the constant of the scoring system.

Table 4 and Fig. 3 show the estimated risk (predicted probabilities) of refusal for ambulatory surgery for each risk score. The estimated risk ranged from $3.855 \%$ for a patient with a score of 0 and $99.979 \%$ for patients with score 17 .

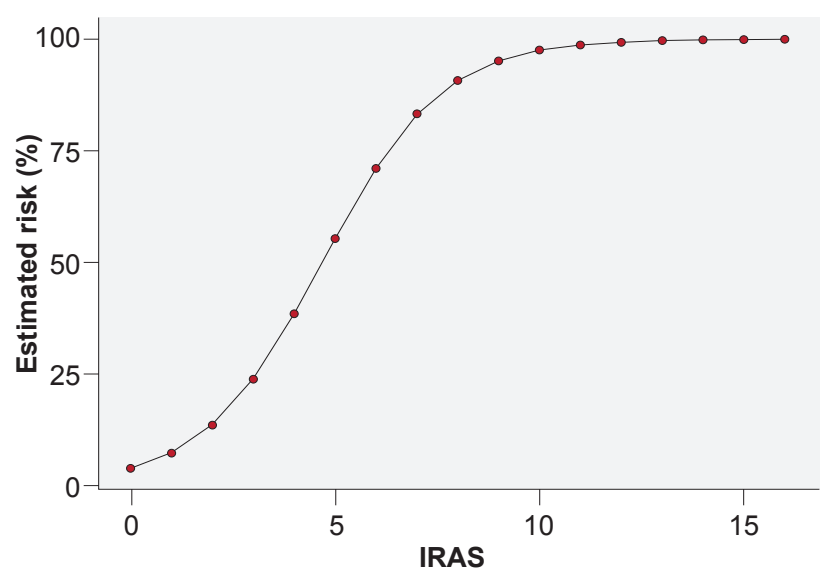

Figure 3 - Estimated probability (estimated risk) of refusal of ambulatory surgery using IRAS 
Table 5 - IRAS associated with refusal of ambulatory surgery for test patients

\begin{tabular}{cccccc}
\hline & \multicolumn{2}{c}{$\begin{array}{c}\text { Refused patients }(n=31) \\
\text { Estimated risk }\end{array}$} & Number of patients & IRAS & \multicolumn{2}{c}{ Non refused patients $(\mathrm{n}=31)$} \\
Estimated risk & Number of patients \\
\hline 0 & 3.855 & 1 & 0 & 3.855 & 16 \\
2 & 13.672 & 1 & 2 & 13.672 & 4 \\
5 & 55.419 & 3 & 3 & 23.939 & 2 \\
6 & 71.185 & 7 & 6 & 71.185 & 1 \\
8 & 90.704 & 5 & 8 & 90.704 & 1 \\
9 & 95.095 & 4 & & & \\
11 & 98.711 & 2 & & & \\
12 & 99.347 & 1 & & \\
14 & 99.833 & 5 & & \\
\hline
\end{tabular}

In order to use the IRAS in clinical practice, a threshold must be defined in order to rule out the refusal for ambulatory surgery. This threshold may be adjusted in order to obtain certain pre-requisites, for example, to obtain a certain positive predictive value (IRAS makes a positive prediction - refusal - and the patient is really refused) or a certain negative predictive value (IRAS makes a negative prediction - no refusal - and the patient isn't refused). We can also choose a threshold to rule out refusal for ambulatory surgery that ensures a certain probability of refusal. For example, defining five points as a threshold - that is, patients who score less than five should be proposed for ambulatory surgery - implies that, for a patient with a predicted risk less than $50 \%$ (Table 4), the model will not refuse him for ambulatory surgery.

The model was tested in a group of randomly chosen 62 patients undergoing abdominal wall hernia surgery during 2019. Within the 62 patients, 31 had been initially refused for ambulatory surgery based on the clinical registries.

Table 5 presents the value of IRAS for the 62 test patients. IRAS ranged from 0 (estimated risk $=3.855 \%$ ) to 11 (estimated risk $=98.711 \%$ ) in the non-refused patient group, and from 0 (estimated risk $=3.855 \%$ ) to 17 (estimated risk $=99.979 \%$ ) in the refused patients group.

A threshold equal to five points meant a positive predictive value (or true positive) of $93.550 \%$ and negative predictive value (or true negative) of $87.100 \%$, as detailed in Table 6.

\section{DISCUSSION}

Outpatient or ambulatory surgery is established nowadays in most western societies as a safe and effective way to perform certain surgical protocols. Its implementation is also advancing in developing countries as well. 8,9

Outpatient surgery allows reduced hospital stays with

Table 6 - Positive and negative predictive values for the model

\begin{tabular}{ccc}
\hline ReallModel & Not refused & Refused \\
\hline Non refused & $87.10 \%$ & $12.90 \%$ \\
Refused & $6.45 \%$ & $93.55 \%$ \\
\hline
\end{tabular}

lower rates of hospital-acquired infections and associated comorbidities and improved cost effectiveness. Overall, and based on the literature, patients are satisfied with outpatient procedures. ${ }^{10,11}$

The progress in terms of anesthetical and minimally invasive surgical protocols has allowed the inclusion of clinically more complex patients in an ambulatory setting in a wider range of medical specialties. Given the technical evolution regarding equipment and procedures, it is expected that this trend will continue in the coming future..$^{12-15}$

Due to the current demand for ambulatory procedures by both patients and institutions, an efficient management of enrolled patients is of utmost importance to ensure the adequate sustainability of the ambulatory surgery process, allowing more patients to be operated with progressively shorter surgery waiting lists.

We observed that a percentage of patients initially proposed for outpatient surgery are rejected and redirected to inpatient care where they have to enrol in another waiting list, which leads to additional morbidity time and loss of active days, until undergoing surgery. This study determined which variables are relevant to the physician in order to reject or accept a patient for ambulatory surgery and developed a mathematical model using data from patients with abdominal wall hernia that predicts the probability of acceptance/rejection of a given patient. Furthermore, we tested the model in a random group of new patients with good results. One should bear in mind, however, that the variables and methodology chosen are applied to abdominal wall hernia. Although the same principles and methods may apply to other conditions, these are valid in this specific context.

This 5-year retrospective analysis shows that the majority of patients refused for ambulatory surgery were declined due to medical reasons. The reasons for this refusal, as described in clinical records and individual registries), are not entirely objective for defining a standard profile of a patient to be rejected.

Due to this observation, a randomly selected group of patients that was referred and underwent abdominal wall hernia surgery in ambulatory setting was used for comparison with the group of rejected patients. After 
analysing both groups, it was observed that patients refused for ambulatory surgery were older, had a higher proportion of cardiovascular risk factors such as high blood pressure, dyslipidemia and type 2 diabetes mellitus. This group also had a significantly higher proportion of atrial fibrillation, ischemic cardiopathy, history of acute coronary syndrome, chronic kidney disease, chronic obstructive pulmonary disease, obstructive sleep apnea under Ci-PAP, and prior abdominal surgery. Finally, they also presented higher ASA Physical Status scores and had a significantly higher use of hypocoagulant and antiplatelet drugs.

Overall, one can state that the rejected patients were older patients with more conditions and more comorbidities and that, although the reasons for refusal are not objectively defined in the medical registries, these are in fact related with the specific pathological conditions and comorbidities. Such a detailed analysis is, to our knowledge, novel considering the current state of the art.

Using logistic regression analysis, a predictive model based on AUROC was built. It considers each individual patient based on his/her profile for the 94 variables studied for the conception of the model and estimates the probability of rejection/acceptance for ambulatory surgery.

Testing of a randomly selected group of patients out of our study group was performed in order to assess the accuracy of the model. A total of 62 patients that underwent abdominal wall hernia surgery during 2019 were randomly selected and evaluated. The model presented a concordance statistic (C-statistic) of 0.86 . The C-statistic for a logistic regression model, a measure of goodness of fit for binary outcomes in a logistic regression model, is a commonly used statistic that measures discrimination. It generally lies between 0.5 and 1 with values close to 0.5 representing poor discrimination between patients (in this case rejected/ accepted) and values closer to 1 representing good discrimination. Based on the literature, our model is considered a strong predictive model. ${ }^{16,17}$ It can be a valid tool to aid in the medical decision of referral of a given patient to either inpatient or ambulatory care. Due to its construction, the input of data on conditions and comorbidities of a specific patient can be converted to a user-friendly tick box algorithm, which instantly delivers a probability of rejection/acceptance. In primary care for example, physicians can consider this information in their decision to direct a patient with an abdominal wall hernia to outpatient or inpatient care. In this way, a more informed decision is made on whether to send a patient, in order to more rapidly and effectively solve his/her medical issue.

Based on the analysed data, a patient proposed for ambulatory surgery that was declined, has a statistically significant additional waiting period of 11 months until surgery (571 \pm 324 days), as compared with a similar patient undergoing surgery in outpatient care $(240 \pm 169$ days $)(p<0.05)$. This represents an added burden in terms of morbidity time and loss of working days. Moreover, a more precise and informed referral might save human and financial resources if one considers the enrolment in medical and nursing appointments, in both outpatient and inpatient scenarios.

There are inherent limitations to this study. Firstly, the study describes the reality of a third line specialised central hospital in Western Europe. The habits and conditions of patients may not necessarily be generalizable to other populations although, in the authors' perspective, the results are expected to reflect the trends at a similar societal level.

Secondly, the model was built based on a 'template' of abdominal wall hernia surgery which constitutes a limitation in terms of broadness. However, this study shows that the reasons for refusal are mostly related with the individual profile of patients rather than the underlying condition itself. Therefore, it is reasonable to assume that its use can be adjusted to other surgical conditions not only within General Surgery but also in other medical specialties where ambulatory surgery is an alternative.

Moreover, the attribution of a level index based on the beta coefficient of the logistic regression model might overlook some aspects related with the clinical value of each particular variable. Finally, some limitations in the clinical application of the current model might be present due to the lack of external validation.

Focusing on future improvements of the healthcare system, strategies to cope with the reasons for refusal of a given patient in an outpatient setting can be described. For example, if one considers obstructive sleep apnea, a protocol and supportive guidelines to be followed can be developed for managing such patients. Ultimately, it would enable their inclusion as successfully treated patients in an ambulatory setting.

\section{CONCLUSION}

We analysed the reasons of refusal of a series of patients in an outpatient setting using five year-retrospective data from patients with abdominal wall hernias. When comparing these with patients that successfully underwent ambulatory surgery because of the same condition, we observed that certain comorbidities and conditions are significantly more prevalent in the refused population. Based on this observation, a mathematical model was developed by means of a logistic regression analysis and successfully tested in a group of new patients. It predicts, with good accuracy, which patients have a higher likelihood of being rejected or accepted for ambulatory surgery. Such a tool can be made accessible by a user-friendly interface that can aid in the decision of referring a specific patient to either inpatient or outpatient care. Indeed, the trend in most western societies is to reinforce ambulatory care in medical institutions while maintaining the possibility for either inpatient or outpatient care. This study can contribute to a reduction in waiting time until surgery, with less morbidity time and improved quality of life for patients, along with added efficacy in terms of healthcare management.

\section{AUTHORS CONTRIBUTIONS}

JO, SR, MC: Conception and draft. Data acquisition and 
analysis. Writing and reviewing the paper. Final approval of the manuscript.

IN, CM, MS: Writing and reviewing the paper. Final approval of the manuscript.

\section{PROTECTION OF HUMANS AND ANIMALS}

The authors declare that the procedures were followed according to the regulations established by the Clinical Research and Ethics Committee of Centro Hospitalar Universitário do Porto and to the Helsinki Declaration of the World Medical Association updated in 2013.

\section{REFERENCES}

1. Nunes J, Gomes R, Povo A, Alves E. Quality indicators in ambulatory surgery: a literature review comparing Portuguese and international systems. Acta Med Port. 2018;31:425-30.

2. Kabré $\mathrm{Y}$, Traoré I, Kaboré $\mathrm{F}$, Bertille $\mathrm{K}$, Traoré $\mathrm{A}$, Ouédraogo I, et al. Anesthesia for ambulatory pediatric surgery in Sub-Saharan Africa: a pilot study in Burkina Faso. Anesth Analg. 2017;124:623-6.

3. Odhiambo M, Njuguna S, Waireri-Onyango R, Mulimba J, Ngugi P. Utilization of day surgery services at Upper Hill Medical Centre and the Karen Hospital in Nairobi: the influence of medical providers, cost and patient awareness. Pan Afr Med J. 2015;22:8.

4. Majholm B, Engbæk J, Bartholdy J, Oerding H, Ahlburg P, Ulrik AM, et al. Is day surgery safe? A Danish multicentre study of morbidity after 57,709 day surgery procedures. Acta Anaesthesiol Scand. 2012;56:323-31.

5. Mathis M, Naughton N, Shanks A, Freundlich R, Pannucci C, Chu Y, et al. Patient selection for day case-eligible surgery: identifying those at high risk for major complications. Anesthesiology. 2013;119:1310-21.

6. Jaensson $\mathrm{M}$, Dahlberg $\mathrm{K}$, Nilsson $\mathrm{U}$. Factors influencing day surgery patients' quality of postoperative recovery and satisfaction with recovery: a narrative review. Perioper Med. 2019;8:3.

7. Verma R, Wee M, Hartle A, Alladi V, Rollin AM, Meakin G, et al. Pre-operative assessment and patient preparation the role of the anaesthetist. London: The Association of Anaesthetists of Great Britain and Ireland; 2010.

8. Mitchell J. Effect of physician ownership of specialty hospitals and ambulatory surgery centers on frequency of use of outpatient orthopedic surgery. Arch Surg. 2010;145:732-8.

9. Zholudev V, Khan A, Patil D, Filson C, Mehta A. Use of office versus

\section{DATA CONFIDENCIALITY}

The authors declare having followed the protocols in use at their working centre regarding patients' data publication.

\section{CONFLICTS OF INTEREST}

The authors have no conflicts of interest to declare.

\section{FUNDING SOURCES}

This research received no specific grant from any funding agency in the public, commercial, or not-for-profit sectors.

ambulatory surgery center setting and associated ancillary services on healthcare cost burden for vasectomy procedures. Urology. 2019;129:29-34

10. Sousa J, Brandão D, Barreto P, Ferreira J, Almeida Lopes J, Mansilha A. Tratamento endovascular do aneurisma da aorta abdominal por via percutânea e anestesia local - one day surgery. Acta Med Port. 2016;29:381-8

11. Lemos P, Pinto A, Morais G, Pereira J, Loureiro R, Teixeira, et al. Patient satisfaction following day surgery. J Clin Anesth. 2009;21:200-5.

12. Quemby DJ, Stocker ME. Day surgery development and practice: key factors for a successful pathway. Continuing Education in Anaesthesia Critical Care Pain. 2014;14:256-61.

13. Vons $\mathrm{C}$. Ambulatory surgery: an evolution of both the surgical technics and the way of care. To excellence in surgery. Presse Med. 2014;43:27882.

14. Gignoux B, Gosgnach M, Lanz T, Vulliez A, Blanchet MC, Frering V, et al. Short-term outcomes of ambulatory colectomy for 157 consecutive patients. Ann Surg. 2019;270:317-21.

15. Sivaganesan A, Hirsch B, Phillips FM, McGirt MJ. Spine surgery in the ambulatory surgery center setting: value-based advancement or safety liability? Neurosurgery. 2018;83:159-65.

16. Hosmer D, Lemeshow S. Applied logistic regression. $2^{\text {nd }}$ ed. New York: John Wiley \& Sons; 2000.

17. Caetano S, Sonpavde G, Pond G. C-statistic: a brief explanation of its construction, interpretation and limitations. Eur J Cancer. 2018;90:1302. 